\title{
On Some New Linear Generating Relations Involving Multivariable H-Function
}

\author{
Dr. Seema Marskole ${ }^{1}$, Dr. S. S. Shrivastava ${ }^{2}$ \\ ${ }^{1}$ Govt. P. G. College Seoni (M. P.) \\ ${ }^{2}$ Institute for Excellence in Higher Education, Bhopal (M. P.)
}

Abstract: The aim of this research paper is to establish some new linear generating relations involving multivariable $H$-function.

\section{Introduction}

The multivariable $\mathrm{H}$-function given in [1] is defined as follows:

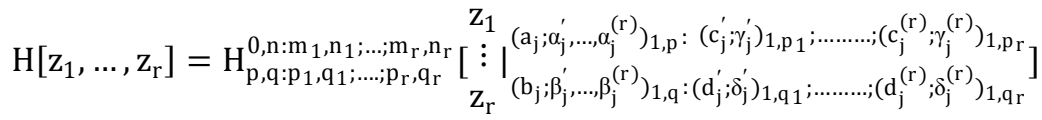

$$
\begin{aligned}
& =\frac{1}{(2 \pi \omega)^{\mathrm{r}}} \int_{\mathrm{L}_{1}} \ldots \int_{\mathrm{L}_{\mathrm{r}}} \phi_{1}\left(\xi_{1}\right) \ldots \phi_{\mathrm{r}}\left(\xi_{\mathrm{r}}\right) \psi\left(\xi_{1}, \ldots, \xi_{\mathrm{r}}\right) \mathrm{z}_{1} \xi_{1} \ldots \mathrm{z}_{\mathrm{r}} \xi_{\mathrm{r}} \mathrm{d} \xi_{1} \ldots \mathrm{d} \xi_{\mathrm{r}}
\end{aligned}
$$

where $\square=\square(1)$,

$$
\begin{aligned}
& \psi\left(\xi_{1}, \ldots, \xi_{r}\right)=\frac{\prod_{j=1}^{n} \Gamma\left(1-a_{j}+\sum_{i=1}^{r} \alpha_{j}^{(i)} \xi_{j}\right)}{\prod_{j=n+1}^{p} \Gamma\left(a_{j}-\sum_{i=1}^{r} \alpha_{j}^{(i)} \xi_{i}\right) \prod_{j=1}^{q} \Gamma\left(1-b_{j}+\sum_{i=1}^{r} \beta_{j}^{(i)} \xi_{i}\right)} \\
& \phi_{i}\left(\xi_{i}\right)=\frac{\prod_{j=1}^{m_{i}} \Gamma\left(d_{j}^{(i)}-\delta_{j}^{(i)} \xi_{j}\right) \prod_{j=1}^{n_{i}} \Gamma\left(1-c_{j}^{(i)}+\gamma_{j}^{(i)} \xi_{i j}\right)}{\prod_{j=m_{i}+1}^{q_{i}} \Gamma\left(1-d_{j}^{(i)}+\delta_{j}^{(i)} \xi_{j}\right) \prod_{j=n_{i}+1}^{p_{i}} \Gamma\left(c_{j}^{(i)}-\gamma_{j}^{(i)} \xi_{i}\right)}
\end{aligned}
$$

In (1), i in the superscript (i) stands for the number of primes, e.g., $b^{(1)}=b^{\prime}, b^{(2)}=b^{\prime \prime}$, and so on; and an empty product is interpreted as unity.

Suppose, as usual, that the parameters

$$
\begin{aligned}
& \mathrm{a}_{\mathrm{j}}, \mathrm{j}=1, \ldots, \mathrm{p} ; \mathrm{c}_{\mathrm{j}}^{(\mathrm{i})}, \mathrm{j}=1, \ldots, \mathrm{p}_{\mathrm{i}} ; \\
& \mathrm{b}_{\mathrm{j}}, \mathrm{j}=1, \ldots, \mathrm{q} ; \mathrm{d}_{\mathrm{j}}^{(\mathrm{i})}, \mathrm{j}=1, \ldots, \mathrm{q}_{\mathrm{i}} ; \square \mathrm{i} \square\{1, \ldots, \mathrm{r}\}
\end{aligned}
$$

are complex numbers and the associated coefficients

$$
\begin{aligned}
& \square_{\mathrm{j}}^{(\mathrm{i})}, \mathrm{j}=1, \ldots, \mathrm{p} ; \square_{\mathrm{j}}^{(\mathrm{i})}, \mathrm{j}=1, \ldots, \mathrm{p}_{\mathrm{i}} ; \\
& \square_{\mathrm{j}}^{(\mathrm{i})}, \mathrm{j}=1, \ldots, \mathrm{q} ; \square_{\mathrm{j}}^{(\mathrm{i})}, \mathrm{j}=1, \ldots, \mathrm{q}_{\mathrm{i}} ; \square \mathrm{i} \square\{1, \ldots, \mathrm{r}\}
\end{aligned}
$$

positive real numbers such that the left of the contour. Also

$$
\begin{gathered}
V_{i}=\sum_{j=1}^{p} \alpha_{j}^{(i)}+\sum_{j=1}^{p_{i}} \gamma_{j}^{(i)}-\sum_{j=1}^{q} \beta_{j}^{(i)}-\sum_{j=1}^{q_{i}} \delta_{j}^{(i)} \leq 0 \\
\Omega_{\mathrm{i}}=-\sum_{\mathrm{j}=\mathrm{n}+1}^{\mathrm{p}} \alpha_{\mathrm{j}}^{(\mathrm{i})}-\sum_{\mathrm{j}=1}^{\mathrm{q}} \beta_{\mathrm{j}}^{(\mathrm{i})}+\sum_{\mathrm{j}=1}^{\mathrm{m}_{\mathrm{i}}} \delta_{\mathrm{j}}^{(\mathrm{i})}-\sum_{\mathrm{j}=\mathrm{m}_{\mathrm{i}}+1}^{\mathrm{q}_{\mathrm{i}}} \delta_{\mathrm{j}}^{(\mathrm{i})}+\sum_{\mathrm{j}=1}^{\mathrm{n}_{\mathrm{i}}} \gamma_{\mathrm{j}}^{(\mathrm{i})}-\sum_{\mathrm{j}=\mathrm{n}_{\mathrm{i}}+1}^{\mathrm{p}_{\mathrm{j}}} \gamma_{\mathrm{j}}^{(\mathrm{i})}>0
\end{gathered}
$$

where the integral $\mathrm{n}, \mathrm{p}, \mathrm{q}, \mathrm{m}_{\mathrm{i}}, \mathrm{n}_{\mathrm{i}}, \mathrm{p}_{\mathrm{i}}$ and $\mathrm{q}_{\mathrm{i}}$ are constrained by the inequalities $\mathrm{p} \square \square \mathrm{n} \square \square 0, \mathrm{q} \square \square \mathrm{q}, \mathrm{q} \square \mathrm{m}$ $\square \square \square$ and $_{\mathrm{i}} \mathrm{p} \square \square \mathrm{n} \square \square \square \square \square \mathrm{i} \square\{1,2, \ldots, \mathrm{r}$ ) and the inequalities $\mathrm{i}$ ) (hold for suitably restricted values of the complex variables $z_{1}, \ldots, z_{r}$. The sequence of parameters in (1) are such that none of the poles of the integrand coincide, that is, the poles of the integrand in (1) are simple. The contour $L_{i}$ in the complex $\square_{i} \square$ plane is of the Mellin-Barnes type which runs from $-\square \square$ to $+\square \square \square$ with indentations, if necessary, to ensure that all the poles of $\left.\square \square \square \mathrm{j}^{(\mathrm{i})} \square \square_{\mathrm{j}}^{(\mathrm{i})} \square_{\mathrm{i}}\right), \mathrm{j}=1, \ldots, \mathrm{m}_{\mathrm{i}}$ are separated from those of $\left.\square \square \square \square \square \square \mathrm{j}^{(\mathrm{i})} \mathrm{c} \square_{\mathrm{j}}^{(\mathrm{i})} \square_{\mathrm{i}}\right), \mathrm{i}=1, \ldots, \mathrm{n}_{\mathrm{i}}$.

In the present investigation we require the following formulae:

From Shrivastava and Manocha [2, p.37],

$$
\begin{aligned}
& (\alpha)_{n}=(\alpha, n)=\frac{\Gamma(\alpha+n)}{\Gamma(\alpha)}, \\
& (1 \square \mathrm{z})^{\square \mathrm{a}}=\sum_{n=0}^{\infty}(a)_{n} \frac{z^{n}}{n !},
\end{aligned}
$$




\section{Linear Generating Relations:}

In this section we establish the following linear Generating Relations:

$$
\begin{aligned}
& \sum_{l=0}^{\infty} \frac{\mathrm{t}^{l}}{l !} \mathrm{H}_{\mathrm{p}, \mathrm{q}:\left(\mathrm{p}_{1}+1, \mathrm{q}_{1}\right) ; \ldots \ldots \ldots \ldots . . ;\left(\mathrm{p}_{\mathrm{r}}, \mathrm{n}_{\mathrm{r}}\right)}^{0, \mathrm{n})} \begin{array}{c}
Z_{1} \\
\vdots \\
Z_{r}
\end{array}
\end{aligned}
$$

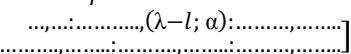

$$
\begin{aligned}
& =(1+\mathrm{t}) \stackrel{(\square \square \square \square}{\mathrm{H}_{\mathrm{p}, \mathrm{q}:\left(\mathrm{p}_{1}+1, \mathrm{q}_{1}\right) ; \ldots \ldots \ldots \ldots ;\left(\mathrm{p}_{\mathrm{r}}, \mathrm{q}_{\mathrm{r}}\right)}^{0, \mathrm{n}}:\left(\mathrm{m}_{1}\right)} \begin{array}{c}
z_{1}(1+t)^{\alpha} \\
\vdots \\
z_{r}
\end{array}
\end{aligned}
$$

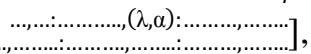

$\mid \arg \left(\left(z_{k}\right) \mid<\frac{1}{2} V_{k} \pi, \forall k \in[1, \ldots, r]\right.$, where $V_{k}$ is given in (2);

$$
\begin{aligned}
& \sum_{l=0}^{\infty} \frac{(-\mathrm{t})^{l}}{l !} \mathrm{H}_{\mathrm{p}, \mathrm{q}:\left(\mathrm{p}_{1}+1, \mathrm{q}_{1}\right) ; \ldots \ldots \ldots \ldots . ;\left(\mathrm{p}_{\mathrm{r}}, \mathrm{q}_{\mathrm{r}}\right)}^{0, \mathrm{n})} \underset{Z_{r}}{Z_{1}}
\end{aligned}
$$

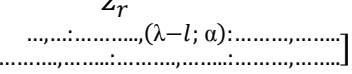

$$
\begin{aligned}
& =(1 \square \mathrm{t})^{(\square \square \square \square} \mathrm{H}_{\mathrm{p}, \mathrm{q}:\left(\mathrm{p}_{1}+1, \mathrm{q}_{1}\right) ; \ldots \ldots \ldots \ldots ;\left(\mathrm{p}_{\mathrm{r}}, \mathrm{q}_{\mathrm{r}}\right)}^{0, \mathrm{n}:\left(\mathrm{m}_{1}, \mathrm{n}_{1}\right) ; \ldots \ldots \ldots \ldots ;}\left[\begin{array}{c}
z_{1}(1+t)^{\alpha} \\
\vdots
\end{array}\right. \\
& \ldots \ldots, \ldots \ldots \ldots \ldots .,(\lambda ; \alpha): \ldots \ldots \ldots, \ldots \ldots \ldots \cdot],
\end{aligned}
$$

$\mid \arg \left(\left(z_{k}\right) \mid<\frac{1}{2} V_{k} \pi, \forall k \in[1, \ldots, r]\right.$, where $V_{k}$ is given in (2).

Proof: To prove (6), consider

$$
\Delta=\sum_{l=0}^{\infty} \frac{\mathrm{t}^{l}}{l !} \mathrm{H}_{\mathrm{p}, \mathrm{q}:\left(\mathrm{p}_{1}+1, \mathrm{q}_{1}\right) ; \ldots \ldots \ldots \ldots ;}^{0, \ldots \ldots ;} \underset{\left(\mathrm{m}_{\mathrm{r}}, \mathrm{n}_{\mathrm{r}}\right)}{\left.\mathrm{q}_{\mathrm{r}}\right)} \begin{gathered}
z_{1} \\
\vdots \\
z_{r}
\end{gathered}
$$

$[\ldots, \ldots \ldots \ldots \ldots \ldots,(\lambda-l ; \alpha): \ldots \ldots \ldots, \ldots \ldots \ldots .$.

On expressing multivariable $\mathrm{H}$-function in contour integral form as given in (1), we get

$$
\Delta=\sum_{l=0}^{\infty} \frac{\mathrm{t}^{l}}{l !}\left[\frac{1}{(2 \pi \omega)^{\mathrm{r}}} \int_{\mathrm{L}_{1}} \ldots \int_{\mathrm{L}_{\mathrm{r}}} \phi_{1}\left(\xi_{1}\right) \ldots . \phi_{\mathrm{r}}\left(\xi_{\mathrm{r}}\right) \psi\left(\xi_{1}, \ldots, \xi_{\mathrm{r}}\right)\right.
$$

$\left.\times \frac{1}{\Gamma\left\{\lambda-l-\alpha \xi_{1}\right\}} z_{1}^{\xi_{1}} \ldots z_{r} \xi_{r} d \xi_{1} \ldots d \xi_{r}\right]$

$$
=\sum_{l=0}^{\infty} \frac{(-\mathrm{t})^{l}}{l !}\left[\frac{1}{(2 \pi \omega)^{\mathrm{r}}} \int_{\mathrm{L}_{1}} \ldots \int_{\mathrm{L}_{\mathrm{r}}} \phi_{1}\left(\xi_{1}\right) \ldots . \phi_{\mathrm{r}}\left(\xi_{\mathrm{r}}\right) \psi\left(\xi_{1}, \ldots, \xi_{\mathrm{r}}\right) .\right.
$$

$\left.\times \frac{\left\{1-\lambda-\alpha \xi_{1}\right\} l}{\Gamma\left\{\lambda-\alpha \xi_{1}\right\}} \mathrm{z}_{1} \xi_{1} \ldots \mathrm{z}_{\mathrm{r}} \xi_{\mathrm{r}} \mathrm{d} \xi_{1} \ldots \mathrm{d} \xi_{\mathrm{r}}\right]$.

On changing the order of summation and integration, we have

$$
\begin{gathered}
\Delta=\frac{1}{(2 \pi \omega)^{\mathrm{r}}} \int_{\mathrm{L}_{1}} \ldots \int_{\mathrm{L}_{\mathrm{r}}} \phi_{1}\left(\xi_{1}\right) \ldots . \phi_{\mathrm{r}}\left(\xi_{\mathrm{r}}\right) \psi\left(\xi_{1}, \ldots, \xi_{\mathrm{r}}\right) \mathrm{z}_{1} \xi_{1} \ldots \mathrm{z}_{\mathrm{r}} \xi_{\mathrm{r}} \\
\times \frac{1}{\Gamma\left\{\lambda-\alpha \xi_{1}\right\}}\left[\sum_{l=0}^{\infty} \frac{(-\mathrm{t})^{l}}{l !}\left\{1-\lambda-\alpha \xi_{1}\right\}_{l}\right] \mathrm{d} \xi_{1} \ldots \mathrm{d} \xi_{\mathrm{r}} \\
=(1+\mathrm{t})^{\lambda-1} \frac{1}{(2 \pi \omega)^{\mathrm{r}}} \int_{\mathrm{L}_{1}} \ldots \int_{\mathrm{L}_{\mathrm{r}}} \phi_{1}\left(\xi_{1}\right) \ldots \phi_{\mathrm{r}}\left(\xi_{\mathrm{r}}\right) \psi\left(\xi_{1}, \ldots, \xi_{\mathrm{r}}\right) \mathrm{z}_{1} \xi_{1} \ldots \mathrm{z}_{\mathrm{r}} \xi_{\mathrm{r}}
\end{gathered}
$$




$$
\times \frac{(1+\mathrm{t})^{\alpha \xi_{1}}}{\Gamma\left\{\lambda-\alpha \xi_{1}\right\}} \mathrm{d} \xi_{1} \ldots \mathrm{d} \xi_{\mathrm{r}}
$$

which in view of (1), provides (6).

Proceeding on similar lines as above, the results (7) can be derived.

\section{Particular Cases}

On specializing the parameters, we get following generating relations in terms of $\mathrm{H}$-function of one variable, which are the results given by Shrivastava \& Shrivastava [3]:

$$
\begin{aligned}
& \sum_{l=0}^{\infty} \frac{(\mathrm{t})^{l}}{l !} \mathrm{H}_{\mathrm{p}+1, \mathrm{q}}^{\mathrm{m}, \mathrm{n}}\left[\left.\mathrm{z}\right|_{\left(\mathrm{a}_{\mathrm{j}}, \mathrm{\alpha}_{\mathrm{j}}\right)_{1, \mathrm{p}},(\lambda-l, \alpha)} ^{\left(\mathrm{b}_{\mathrm{j}}, \beta_{\mathrm{j}}\right)_{1, \mathrm{q}}}\right] \\
& =(1+\mathrm{t})^{(\square \square \square} \mathrm{H}_{\mathrm{p}+1, \mathrm{q}}^{\mathrm{m}, \mathrm{n}}\left[\left.\mathrm{z}(1+\mathrm{t})^{-\alpha}\right|_{\left(\mathrm{p}_{\mathrm{j}}, \beta_{\mathrm{j}}\right)_{1, \mathrm{q}}} ^{\left(\mathrm{a}_{\mathrm{j}}, \mathrm{\alpha}_{\mathrm{j}}\right)_{1, \mathrm{p}},(\lambda, \alpha)}\right],
\end{aligned}
$$

$|\operatorname{argz}|<1 / 2 \mathrm{~A} \square$, where A is givenby

$$
\begin{aligned}
& \sum_{\mathrm{j}=1}^{\mathrm{n}} \alpha_{\mathrm{j}}-\sum_{\mathrm{j}=\mathrm{n}+1}^{\mathrm{p}} \alpha_{\mathrm{j}}+\sum_{\mathrm{j}=1}^{\mathrm{m}} \beta_{\mathrm{j}}-\sum_{\mathrm{j}=\mathrm{m}+1}^{\mathrm{q}} \beta_{\mathrm{j}} \equiv \mathrm{A}>0 ; \\
& \sum_{l=0}^{\infty} \frac{(-\mathrm{t})^{l}}{l !} \mathrm{H}_{\mathrm{p}+1, \mathrm{q}}^{\mathrm{m}, \mathrm{n}}\left[\mathrm{zl}{\left.\stackrel{\left(\mathrm{a}_{\mathrm{j}}, \alpha_{\mathrm{j}}\right)_{1, \mathrm{p}},(\lambda-l, \alpha)}{\left(\mathrm{b}_{\mathrm{j}}, \beta_{\mathrm{j}}\right)_{1, \mathrm{q}}}\right]}^{(1 \square \mathrm{t})^{(\square \square \square} \mathrm{H}_{\mathrm{p}+1, \mathrm{q}}^{\mathrm{m}, \mathrm{n}}\left[\left.\mathrm{z}(1-\mathrm{t})^{-\alpha}\right|_{\left(\mathrm{a}_{\mathrm{j}}, \alpha_{\mathrm{j}}\right)_{1, \mathrm{p}},(\lambda, \alpha)} ^{\left(\mathrm{b}_{\mathrm{j}}, \beta_{\mathrm{j}}\right)_{1, \mathrm{q}}}\right],}\right.
\end{aligned}
$$

$|\operatorname{argz}|<1 / 2 \mathrm{~A} \square$, where $\mathrm{A}$ is given by

$$
\sum_{\mathrm{j}=1}^{\mathrm{n}} \alpha_{\mathrm{j}}-\sum_{\mathrm{j}=\mathrm{n}+1}^{\mathrm{p}} \alpha_{\mathrm{j}}+\sum_{\mathrm{j}=1}^{\mathrm{m}} \beta_{\mathrm{j}}-\sum_{\mathrm{j}=\mathrm{m}+1}^{\mathrm{q}} \beta_{\mathrm{j}} \equiv \mathrm{A}>0 .
$$

\section{References}

[1] Srivastava, H. M., Gupta, K. C. and Goyal, S. P.: The H-function of one and two variables with applications, South Assian Publishers, New Delhi, 1982

[2] Shrivastava, H. M. and Manocha, H. L.: A treatise on generating functions, Ellis Horwood Limited England.

[3] Shrivastava, Shweta and Shrivastava, B. M. L.: Some new generating relations and identities for H-function, Vijnana Parishad Anusandhan Patrika, Vol. 49, No.1, January, 2006, p. 63-77. 